\title{
An Algorithm for Automatic Base Station Placement in Cellular Network Deployment
}

\author{
István Törös and Péter Fazekas \\ High Speed Networks Laboratory \\ Dept. of Telecommunications, Budapest University of Technology and Economics, \\ Magyar tudósok körútja 2., 1117 Budapest, Hungary \\ \{toros,fazekasp\}@hit.bme.hu \\ http://www.springer.com/lncs
}

\begin{abstract}
The optimal base station placement is a serious task in cellular wireless networks. In this paper we propose a method that automatically distributes the base stations on a studied scenario, maintaining coverage requirement and enabling the transmission of traffic demands distributed over the area. A city scenario with normal demands is examined and the advantages/disadvantages of this method are discussed. The planner and optimizing tasks are based on an iterative K-Means clustering method. Numerical results of coverage, as well as graphical results permit on radio coverage maps, are presented as base for main consequences. Furthermore, the final results are compared to another simple planning algorithm.
\end{abstract}

Keywords: cellular network planning, coverage, capacity.

\section{Introduction}

The radio planning of cellular wireless network is a highly investigated topic, because operators can save budget using a cost efficient planning method. The performance of a mobile network is mainly characterized by the received signal strength and signal to noise plus interference ratio at the mobile's position, hence the coverage of the network. The coverage is mainly affected by transmit power and radio propagation, hence depends on the environment of network. Gain of better coverage and increased signal to noise plus interference ratio (SINR) in a cellular network are the key objectives of mobile networking industry. Developing and using an algorithm that automatically plans the positions of base stations and provides the necessary coverage and capacity over the area with the least number of stations, is thus of utmost importance.

Our research is focused on the development and investigation of such an automatic planning algorithm. The frequency adaptation and power control are very simple in our simulation model. Any other, more complex algorithms might be used with our planning method, these would certainly result in lower number of base stations.

The key object of the planning of the new generation networks is that the map of SINR suits to user's distribution. In the position of the users with high 
demand should be provided with high SINR. We could achieve this criterion if the base stations are planned near the high demand users.

This new algorithm is based on a clustering method which is very popular suggestion for planning, the K-means algorithm. However, most of the methods use on K-means concentrate either on dimensioning or optimization and they require prediction of number of beginning clusters, which is not straightforward, see e.g. [1][2]. In [7], the author has used Set Cover Base Station Positioning Algorithm for BS planning. This algorithm is focussed on optimal coverage of networks. The full serving of users by placement algorithm is secondary, because the verification of SINR runs after BS placement. In [6], the authors have focussed attention on special K-means clustering with fixed number of cluster. This may produce good area coverage and have a small amount of overlap between cells. However, such a network will probably not be able to satisfy the traffic demands within each cell. In contrast, our method does both the dimensioning and optimization steps and does not require initial estimations, rather can start with an empty (in terms of number of base stations) area, with an arbitrarily placed single base station and places the necessary stations over this. However, if required, the algorithm might be used starting with an initial arbitrary network topology (location of arbitrary number of base stations) and places new base stations to fulfill coverage and capacity requirements. This is useful in the case when network deployment strategy has to be planned in order to serve increasing capacity demands in an already running network. It is important to note that the location algorithm creates the clusters based on the properties of base station which were initialized at the beginning.

\section{Modelling Environment}

Our goal is to investigate the capability of the new algorithm in mobile networks and evaluate coverage, SINR and quality of transmission in the network. In order to achieve our goal, we used our own developed software which simulates a mobile network, where base stations can be added easily. The model of the environment and the network implemented in the simulator is detailed in the following.

Since the communication range is an important property, the distances between nodes are displayed precisely on the graphic screen. The signal power and SINR are also shown on the screen with color spectrum changed gradually from weak to strong. Based on the signal power, we can calculate the coverage area of a given cell. The SINR parameter guides us to derive an estimated overall performance of the network.

We assume that the network to be developed by the algorithm (and the software tool implementing it) is the novel 3GPP LTE (Long Term Evolution) network. However, the method can be applied to any other radio network, even to subsequent ones (e.g. LTE Advanced). The algorithm assumes that a Radio Resource Control (RRC) mechanism is present that allocates radio resources to different cells according to arbitrary rules. In LTE, this practically means that RRC allocates Physical Resource Blocks (PRB) to cells (a PRB is 12 OFDM subcarriers transmitted in a $1 \mathrm{~ms}$ subframe). 


\subsection{Simulated Terrain}

The environment of simulations is composed of three different layers. The first is the flat geographical layer which helps the speed of simulation. The second is the layer of buildings and roads. In the following, we assume that this is a large city with big houses, but other terrain types are also applicable. For the numerical results presented below, we assumed a $9 \mathrm{~km}^{2}$ city area. The roads are generated by an algorithm that creates first the road network with confluence. The buildings will be placed near the roads with random height. The last is the layer of demands which is created according to the following scheme.

Traffic demands are given per unit area in bits per second (practically: per pixel of the map). If the position is near a road or a confluence then demand of this place will be higher, the demand is function of distance from the road. The model assumes that more traffic is demanded within buildings as well, as in reality high rate applications are used at home or in office, rather than outdoor. In the calculations presented below, the aggregate required bit rate is $900 \mathrm{Mb} / \mathrm{s}$ for the entire map.

\section{$2.2 \quad$ Antenna Model}

To keep the model realistic, sectorized antennas are assumed. The antenna horizontal characteristic is described by equations (1) and (2),

$$
\begin{gathered}
\text { IF } \alpha \leq 90, \text { then Power }=\cos ^{2}(\alpha) * p w . \\
\text { IF } \alpha>90, \text { then Power }=0
\end{gathered}
$$

where $\alpha$ is an angle between the main direction of sector antenna and a changing vector pointing towards the actual location under examination and $p w$ is the transmitter gain extended by antenna gain. Hence, during the calculations, signal strength is determined (along with the path loss model) according to the direction of a given point of the map.

The vertical characteristic is described by (3).

$$
\text { Power }=\cos ^{2}(\alpha-x) * p w .
$$

We can employ (3) in all directions where $\alpha$ is the vertical angle of the main direction of sector antenna and $x$ is the vertical angle of changing vector. The BS-s are planned with the traditional layout, namely three sector antennas with 120 degrees separation between their main directions.

\subsection{Propagation Models}

We use COST 231 path loss model for big city environment in our simulations. This has the advantage that it can be implemented easily without expensive geographical database, yet it is accurate enough, captures major properties of propagation and used widely in cellular network planning. This model is a function of the carrier frequency $f$ which range from $50 \mathrm{MHz}$ to $2 \mathrm{GHz}$, of the 


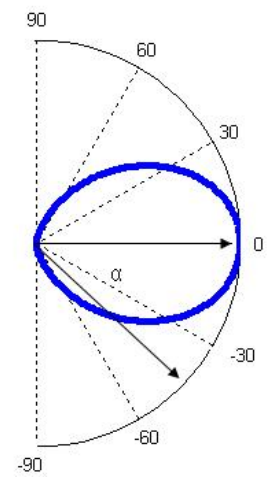

Fig. 1. Horizontal characteristic of Sector Antenna

effective height of base station $h_{b}$ and the effective height of mobile $h_{m}$ [5]. The attenuation is given by equation (4)

$$
\begin{aligned}
A_{p} & =46.3+33.9 * \log _{10} f-13.82 * \log _{10} h_{b}+\left(44.9-6.55 * \log _{10} h_{b}\right) \\
& * \log _{10} d-a\left(h_{m}\right)+C_{m}
\end{aligned}
$$

where for large cities and $f \geq 400 \mathrm{MHz}$.

$$
a\left(h_{m}\right)=3.2 *\left(\log _{10}\left(11.75 * h_{m}\right)\right)^{2}-4.97
$$

and $C_{m}=3 \mathrm{~dB}$ in large cities. Along with this model, a slow fading is also taken into account by means of a single fading margin expressed in $\mathrm{dB}$.

\section{Description of the Algorithm}

\subsection{K-means Clustering}

The method is based on the K-means clustering algorithm that is briefly presented in this section. This is a dynamic clustering which attempts to directly decompose the data into disjoint clusters. We use this algorithm to cluster the demands and form sets of them (cells). The criterion function $(\rho(\mathrm{x}, \mathrm{y}))$ which has to be minimized, is the distance between a given location of demand within the sector $(x)$ and the position of serving base station $(y)$ weighted by the demand of $x$.

The first is the assignment step. Join each demand to the closest cluster.

$$
C_{i}^{t}=\left\{x_{j}: \rho\left(x_{j}, m_{j}\right)<\rho\left(x_{j}, m_{i}^{*}\right) \text { for all } i^{*}\right\}
$$

where $x_{j}$ is the location of demand, $m_{i}$ is the centroid of the $i^{\text {th }}$ cluster (the position of serving base station). $C_{i}^{t}$ is the closest cluster of $x_{j}$ demand at the $t^{t h}$ step. The other is update step. 


$$
m_{i}^{(t+1)}=\frac{1}{\# C_{i}^{t}} \sum_{x \subset C_{i}^{t}} x_{j}
$$

where $\# C_{i}^{t}$ is the number and $x$ is the location of demand within $i^{t h}$ cluster $\left(C_{i}\right)$. This equation (7) calculates the new means to be the center point in the cluster.

\subsection{Input and Output Parameters}

The input parameters of simulation are the two dimension map that includes positions of roads and buildings, the distribution of traffic demands, the available bandwidth, as well as properties of base stations (power, antenna parameters). The output of the algorithm is the locations of the base stations that are needed to provide coverage and serve all traffic demands. Along with this and the information on available spectrum and traffic demands, the frequency utilization and the spectral efficiency of the network are also main outputs of the algorithm.

\subsection{The Planning Algorithm}

Our planning algorithm can be realized as a closed loop (Figure 2). The location algorithm waits for the input parameters, and the result of Radio Resource Control which includes allocation of radio resources (PRBs) to traffic demands. As the main contribution of this paper is the base station location algorithm (LA), the applied RRC in the subsequent analysis is supposed to be a very simple, basic operation. Namely, in the evaluations below the available band (20 $\mathrm{MHz}, 100 \mathrm{PRBs}$ in LTE) is divided to three non-overlapping regions, allocated to the three sectorized cells of each base station. This simple RRC sends to location algorithm the status of service. If a sector is overloaded (the demands in its coverage cannot be served with the fixed band available), then the LA has to eliminate this problem and tries to serve the given area with more stations. This cycle will run until all demands are served.

At the beginning, the initial parameters are fed to the algorithm, practically from a database and a single base station is placed into the center of the area. In the next step, the received signal power from each antenna to every position at the map is calculated. The calculation of received signal power is based on equation (8)

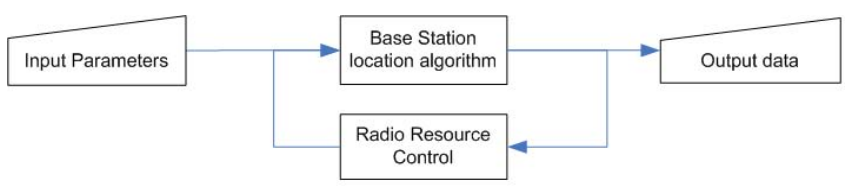

Fig. 2. Main flowchart diagram of RF planning algorithm 


$$
R S=T S+\text { TAgain }-P L+R \text { Again }-C
$$

where $R S$ is the received signal in $\mathrm{dBm}, T S$ is the transmitter power in $\mathrm{dB}$, TAgain is the gain of transmitter antenna in $\mathrm{dB}$ (taking into account antenna characteristics), $P L$ is the pathloss in $\mathrm{dB}$ (COST-231), RAgain is the gain of receiver antenna in $\mathrm{dB}, C$ is the fading margin in $\mathrm{dB}$. This calculation is executed for every position of the map from all antennas. After this procedure we can run the RRC mechanism. The "best server" policy is followed within the network. First of all we have to search the highest value of received signal at every position and note this value and origin of this signal (antennaID). This task is followed the calculation of other cell's interference. At last we can compute value of SINR using (9).

$$
S I N R=\frac{\operatorname{maxSignal}}{\text { Interference }+ \text { Noise }}
$$

The power of thermal noise is taken to be $-101 \mathrm{dBm}$ in the evaluations. The algorithm assigns every location (position of demand) to the sector that will serve it. In the following step we will predict the required bandwidth (number of PRBs) for each sector. The relationship between SINR and required frequency for serving a given demand is based on the so called Alpha-Shannon Formula, suggested to be used for LTE networks (10)[4]

$$
\text { SpectralEfficiency }=\alpha * \log _{2}\left(1+10^{\frac{S I N R}{10 * i m p f a c t o r}}\right)
$$

where $\alpha=0.75$, imp factor $=1.25$, and $S I N R$ is Signal Noise Interference ratio at the actual point in $\mathrm{dB}$. The number of required frequency bands per sector is thus given by (11)

$$
\sum_{\text {point of sector }} \frac{\text { demand }}{\text { SpectralEfficiency }}
$$

After this step it turns out that the sector is able to serve its actual demands or not. The following steps belong to the LA. Searches the "most unserved" sector (MUS), where the absence of required frequency is the highest. If no such sector is found (all demands are served), than the algorithm will terminate. Otherwise the algorithm locates a new base station near the serving antenna of MUS in the main direction. This new BS has to have same parameters as the BS of MUS. The following step is the running of K-means clustering algorithm. The necessary $\rho(\mathrm{x}, \mathrm{m})$ metric is the distance between a position of covered demand $(x)$ within the sector and the position of serving base station $(m)$ weighted by the demand of $x$. The demands of MUS will connect with the new placed BS, because the new signals will arrive from nearer position. The update step of K-Means will shift them (Figure 3). After some cycles the moving of BSs will decrease, hence we intuitively chose the K-means clustering to run for six cycles.

The detailed flowchart diagram of the algorithm is presented in Figure 4. 


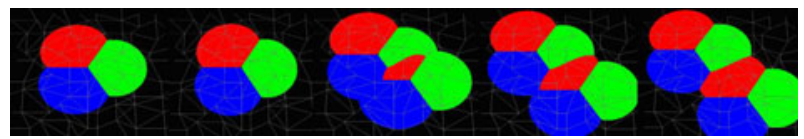

Fig. 3. Mechanism of Location Algorithm

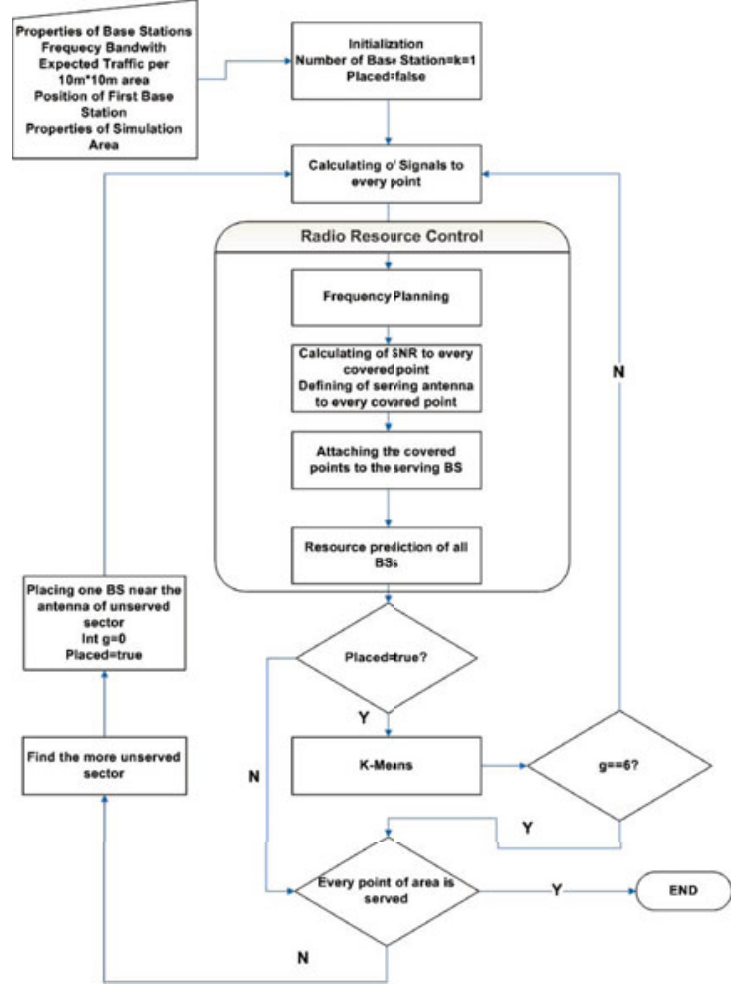

Fig. 4. Detailed flowchart diagram of RF planning algorithm

\section{Numerical Results}

The algorithm was used in a terrain of $9 \mathrm{~km}^{2}$, which models a center of a big city. The overall traffic demand was $900 \mathrm{Mbps}$, the available frequency bandwidth is $20 \mathrm{MHz}$. The Frequency Reuse Factor is 3, therefore one sector uses $6.67 \mathrm{MHz}$ frequency bandwidth.

In Figure 5 the total amount of used and unused frequency bands is presented, as the algorithm placed more and more base stations over the area. The amount of total used and unused frequency is determined by means of summing up all used (and unused) bandwidths for all cells in the network. We can see that the used total bandwidth is reaching its maximum for about 30 base stations (meaning that most of the demands are served at this point), but 10 more base 


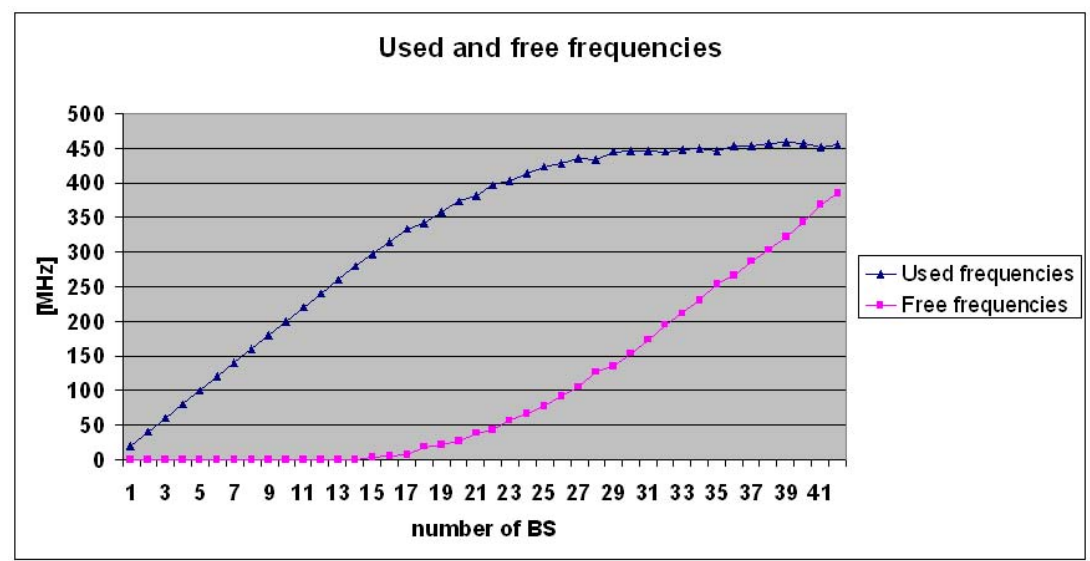

Fig. 5. Used and free frequency band during the running of planning algorithm

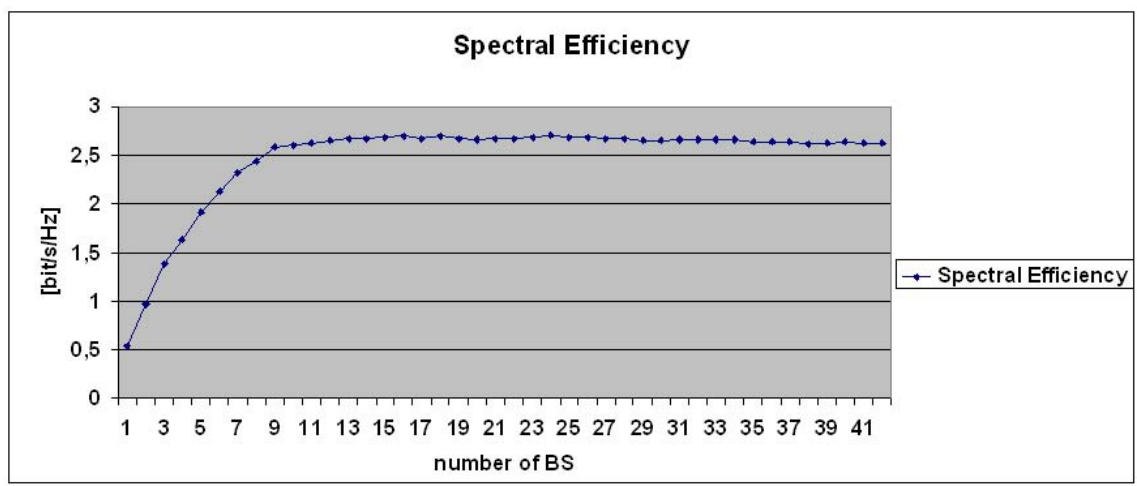

Fig. 6. Spectral efficiency during the running of planning algorithm

stations are needed to attain $100 \%$ coverage and service. This then results in the increase of unused bandwidth, meaning that the service of the last couple of percentage of demands seriously decrease efficiency. The consequences of this are that a given reasonable service requirement (in terms of coverage and capacity), say $95 \%$ (meaning that $5 \%$ of the area might remain uncovered and $5 \%$ of the demands might not be served) can save a lot in terms of required base station numbers. On the other hand, if more advanced RRC would be assumed and modelled (that allocates PRBs adaptively to demands), the efficiency of used spectrum would be higher, thus less base stations would be enough (however, the aim of this work is not to develop an optimal RRC method, but to plan the network with any given RRC).

In Figure 6, the spectral efficiency of the network is presented, as the algorithm places new base stations over the area. It reaches its peak quite early, for about 


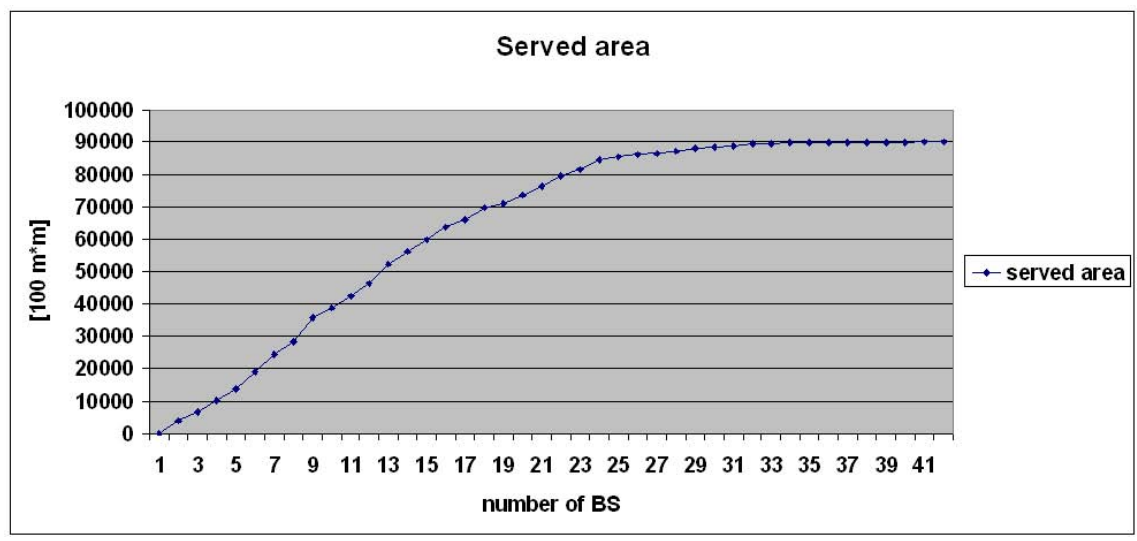

Fig. 7. Size of served area during the running of planning algorithm

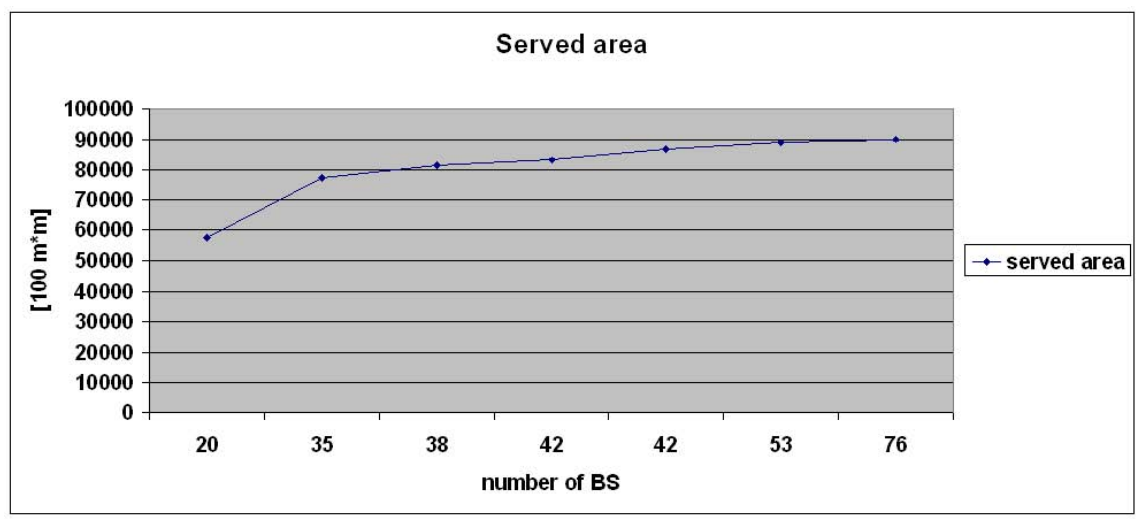

Fig. 8. Size of served area for different hexagonal BS location

13 base stations. This means that the placement of new stations will not increase spectral efficiency, however to serve all demands (and to provide more capacity), more stations are needed. A slight decrease in spectral efficiency can also be observed in case of higher numbers of base stations, that is for the same reasons that was concluded regarding Figure 5 .

Figure 7 shows how the served area is increasing over the iterations of the algorithm. Again, it can be concluded, that the area of $9 \mathrm{~km}^{2}$ is well served by around 30 stations, the rest are needed to cover negligible holes in coverage.

The algorithm proposed was compared to the results of a traditional base station placement following a regular hexagonal grid. Figure 8 shows how the served area increases as the number of base stations increase in this regular grid (We created two different hexagonal grids with $42 \mathrm{BSs}$ ). We can conclude that our proposed algorithm covers the area with half the number of base stations, that is a very reasonable performance increase. 


\section{Conclusions}

In this paper a novel algorithm was shown, that enables the automatic placement and determination of the number of base stations, that can serve a cellular network area with given traffic conditions. The algorithm is based on realistic assumptions an can be used for any legacy system, with arbitrary Radio Resource Control method applied in the network. Numerical methods were presented, showing that the algorithm reaches total coverage and allows the service of all traffic demands, although it is concluded that by means of loosening coverage/capacity requirements, or by using advanced RRC algorithms, the number of necessary base stations might be significantly reduced.

\section{References}

1. Karam, O.H., Fattouh, L., Youssef, N., Abdelazim, A.E.: Employing Clustering Techniques in Planning Wireless Local Loop Communication Systems: PlanAir. In: 11th International Conference on Artificial Intelligence Applications Cairo, Egypt, February 23-26 (2005)

2. Ajay R. Mishra, Advanced Cellular Network Planning and Optimization, pp. 15197,John Wiley \& Sons Ltd. (2007)

3. Glisic, S.G.: Advanced Wireless Networks 4G, pp. 667-694. John Wiley \& Sons Ltd., Chichester (2006)

4. Basit, A.: Dimensioning of LTE Network, Description of Models and Tool, Coverage and Capacity Estimation of 3GPP Long Term Evolution radio interface (2009)

5. Barclay, L.: Propagation of Radiowaves, p. 194. The Institution of Electrical Engineers, London (2003)

6. Ramamurthy, H., Karandikar, A.: B-Hive: A cell planning tool for urban wireless networks. In: 9th National Conference on Communications (2003)

7. Tutschku, K.: Demand-based Radio Network Planning of Cellular Mobile Communication Systems. In: INFOCOM 1998, pp. 1054-1061 (1998) 